\title{
Mutação no gene BRAF em carcinoma diferenciado de tireoide avançado: um relato de caso
}

\author{
Mutation in the BRAF gene in advanced differentiated thyroid carcinoma: a case report
}

\author{
Juliana Lima Von Ammon ${ }^{1 *}$, Helton Estrela Ramos²
}

'Bióloga. Mestranda do Programa de Pós-graduação Processos Interativos dos Órgãos e Sistemas. Instituto de Ciências da Saúde. Universidade Federal da Bahia - UFBA. ${ }^{2}$ Médico. Professor Associado. Chefe do Laboratório de Estudo da

Tireoide. Instituto de Ciências da Saúde - UFBA

\begin{abstract}
Resumo
Introdução: o carcinoma diferenciado da tireoide corresponde aproximadamente a $90 \%$ dos casos de neoplasias da tireoide, sendo que, desses, $80-85 \%$ dos casos são de carcinoma papilífero da tireoide. Apesar de a maioria desses carcinomas serem bem diferenciados e com baixa taxa de invasão local, recidiva ou metástases (regionais ou distantes), existe um subgrupo que apresenta uma heterogeneidade genética com variantes mais agressivas, podendo tornar o câncer de tireoide mais invasivo e letal. Objetivos: 1) Relatar o caso de um paciente adulto portador de carcinoma diferenciado da tireoide avançado, com metástase pulmonar, em terapia supressiva com levotiroxina, após tratamento combinado com cirurgia e radioiodoterapia; 2) Discutir sobre as características patológicas e moleculares como fatores relevantes no direcionamento de condutas terapêuticas. Caso clínico: trata-se de um paciente do sexo masculino, com 39 anos de idade, cuja a punção aspirativa com agulha fina (PAAF) foi compatível com carcinoma papilífero da tireoide que, na sequência, foi submetido a tireoidectomia total, com esvaziamento cervical. O exame anatomopatológico revelou carcinoma papilífero da tireoide clássico, com metástase em cinco linfonodos. A análise molecular realizada por sequenciamento de nova geração evidenciou a mutação no gene $B R A F$ (V600E), o qual tem associação significativa com estágios mais avançados do tumor, metástases e radioiodorefratariedade. Conclusão: este relato possibilita discutir a necessidade da avaliação molecular como direcionamento na conduta terapêutica de cada paciente oncológico. Palavras-chave: Câncer Papilifero da Tireoide. Proteínas Proto-Oncogênicas B-raf. Sequenciamento de Nova Geração.
\end{abstract}

\begin{abstract}
Introduction: differentiated thyroid cancer accounts for approximately $90 \%$ cases of thyroid neoplasms, and among them $80-85 \%$ of cases are papillary thyroid carcinoma. Although most of this type of carcinoma is well differentiated and has a low rate of local invasion, recurrence, or metastases (regional or distant), there is a subgroup of tumors that present genetic heterogeneity with more aggressive variants, which may make thyroid cancer more invasive and lethal. Objectives: 1) To report the case of an adult patient with advanced differentiated thyroid carcinoma, and pulmonary metastasis, undergoing suppressive therapy with levothyroxine, after combined treatment with surgery and radioiodine therapy; 2) Discuss pathological and molecular characteristics as relevant factors in directing therapeutic approaches. Case report: a 39-year-old male patient, whose fine needle aspiration biopsy (FNAB) was compatible with papillary thyroid carcinoma, who subsequently underwent total thyroidectomy with neck dissection. Pathological examination revealed classic papillary thyroid carcinoma, with metastasis in five lymph nodes. Molecular analysis performed by second generation sequencing showed a mutation in BRAF gene (V600E), which has a significant association with more advanced stages of the tumor, metastases and radioiodine refractoriness. Conclusion:tThis report makes possible to discuss the need for molecular assessment as a guideline on therapeutic conduct of each cancer patient.

Keywords: Papillary thyroid cancer. Proto-oncogene proteins B-raf. Next-generation sequencing.
\end{abstract}

\section{INTRODUÇÃO}

O câncer de tireoide (CT) representa a malignidade endócrina mais comum, sendo responsável por $3,6 \%$ de todos os cânceres diagnosticados anualmente'.

O carcinoma diferenciado da tireoide (CDT) corresponde, aproximadamente, a $90 \%$ dos casos de neoplasias da tireoide e refere-se às derivadas de células foliculares, incluindo câncer papilar de tireoide (CPT), câncer folicular

Correspondente/Corresponding: *Juliana Lima Von Ammon - Instituto de Ciências da Saúde, Universidade Federal da Bahia - End.: Av. Reitor Miguel Calmon, s/n, Vale do Canela 40. 110-100 - Salvador - BA - Tel: (71) 99207-1942 -E-mail: juliana.ammon@ufba.br da tireoide (CFT) e câncer de células de Hurthle. O CPT corresponde a $80-85 \%$ dos casos ${ }^{2,3}$. Geralmente, ele é clinicamente indolente, compatível com seu genoma simples, apresentando poucas alterações no número de cópias². Embora tenha uma alta incidência, apresenta taxas de sobrevida global excedendo a $90 \%^{3,4,15}$. Apesar de a maioria dos CPT ser bem diferenciada e com baixa taxa de invasão local, recidiva ou metástases (regionais ou distantes), existe em subgrupo de tumores que apresenta heterogeneidade genética associada com variantes histológicas de maior agressividade, podendo tornar o CDT mais invasivo e letal ${ }^{5}$

As características clínicas e histológicas não predizem precisamente o comportamento biológico dos tumores de tireoide, sendo relevante identificar, por meio de exames 
moleculares, os pacientes com maior risco de agressividade da doença, a fim de prevenir resultados desfavoráveis e implementar terapias mais personalizadas 6 .

A maioria das mutações envolvidas na tumorigênese tireoidiana envolve os efetores das vias MAPK ou PI3K-AKT, que resultam em uma desregulação do crescimento celular e diferenciação ${ }^{7}$. A ativação de MAPK é considerada crucial para a iniciação de CPT, por meio de mutações pontuais dos genes BRAF e RAS ou fusões gênicas de RET / PTC $e$ $N T R K^{8,14}$. Essas mutações ocorrem em aproximadamente $70 \%$ dos casos de CPT ${ }^{7,13}$.

A mutação somática de maior evidência no CDT encontra-se no gene $B R A F$, que resulta em uma transversão de timina para adenina no nucleotídeo 1799 (T1799A), levando a uma substituição de valina por ácido glutâmico no resíduo 600 da proteína (V600E) 7,9. Mutação no gene $B R A F$ tem uma associação significativa com estágios mais avançados do tumor, promovendo sua invasividade, metástase e recorrência, além de mostrar uma baixa resposta ao tratamento com radioiodo, sendo assim um importante fator prognóstico em $\mathrm{CPT}^{2,10,12,15}$.

O tratamento padrão do CPT é intervenção cirúrgica e terapia de ablação com radioiodo pós-operatório. $O$ transporte de iodo para tireoide é mediado pelo simportador sódio-iodo (NIS), uma transmembrana glicoproteína normalmente localizada na membrana basolateral das células foliculares da tireoide. Pacientes com excelentes respostas a radioiodoterapia (RT) têm bom prognóstico e baixa taxa de recorrência. Porém, aqueles resistentes à RT apresentam um maior risco de recorrências locais e metástases, podendo resultar em uma maior taxa de mortalidade ${ }^{11}$.

Neste artigo, um estudo observacional do tipo descritivo, será relatado o caso de um paciente adulto portador de CDT avançado, com metástase pulmonar e mutação V600E do gene BRAF na análise tumoral. O paciente foi atendido na Clínica IT - Endocrinologia Especializada Ltda, bem como se submeteu a tratamento combinado com tireoidectomia total (TT) e radioiodoterapia, encontrando-se atualmente em terapia supressiva com levotiroxina. Além disso, este texto revisa a literatura a respeito das características moleculares do CDT avançado e discute as modalidades terapêuticas.

\section{CASO CLÍNICO}

Paciente do sexo masculino, 39 anos de idade, foi diagnosticado com CDT, no ano de 2019, submetido a duas cirurgias, sendo a última para maior esvaziamento de compartimentos cervicais.

O laudo anatomopatológico revelou CPT clássico, não encapsulado, multifocal, localizado no LD, LE e istmo

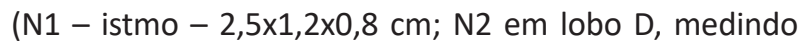
$0,8 \times 0,7 \times 0,5 \mathrm{~cm}$; N3 em lobo esquerdo, medindo $0,3 \times 0,2$ $\mathrm{cm})$. Havia comprometimento da superfície tireoidiana, com extensão extratireoidiana (ETE) mínima para tecido adiposo, sem invasão vascular sanguínea, com invasão vascular linfática e sem invasão perineural; 5 de 9 linfo- nodos foram acometidos, mas não havia ruptura capsular,

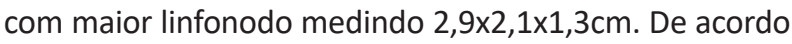
com o sistema de estadiamento patológico TNM (tumor, nódulo e metástase), a oitava edição do American Joint Committee on Cancer (AJCC ${ }^{21}$ classifica esse caso como de estágio II, pois é pT2m (tumor primário multifocal, medindo entre $2 \mathrm{~cm}$ a $4 \mathrm{~cm}$ ), N1b (apresenta metástase em outros compartimentos cervicais unilaterais, bilaterais ou contralaterais - níveis I, II, III, IV ou V - ou retrofaríngeos) e M1 (metástase à distância). Foi classificado como alto risco de recorrência, de acordo com os critérios estabelecidos pela American Thyroid Association (ATA) ${ }^{22}$.

Após a tireoidectomia total com esvaziamento cervical, o paciente foi submetido à radioiodoterapia em março de 2019 e à pesquisa de corpo inteiro ( $\mathrm{PCl}$ ) pós-tratamento, no qual obteve captação satisfatória da dose terapêutica com iodo-131 na região cervical, com ausência de captação em pulmões (Figura 1).

Figura 1 - PCl com lodo-131. Primeira imagem à esquerda foi realizada antes de 48 horas após a dose, em seguida imagem após 48 horas. Terceira imagem à direita mostra a região cervical com bastante captação do lodo-131

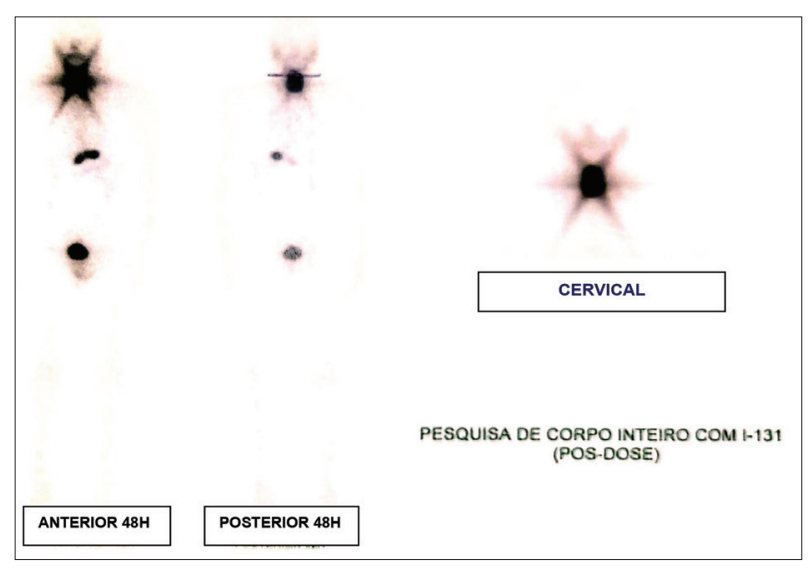

Fonte: Dados da pesquisa.

Em janeiro de 2020, o paciente realizou a tomografia computadorizada por emissão de pósitrons (PET-CT) e foi evidenciada persistência da doença, caracterizada pela presença de múltiplos nódulos, com atenuação de partes moles, hipermetabólicos, medindo até $0,6 \mathrm{~cm}$; o valor de absorção padrão (SUV), que expressa a atividade metabólica da lesão, foi de 9,4 máximo, com distribuição aleatória esparsa em ambos os pulmões; também foi descrita linfonodomegalia hipermetabólica em cadeia paratraqueal superior direita, medindo $1,1 \mathrm{~cm}$ no menor eixo axial, com SUV máximo 21 (Figura 2). Exames laboratoriais realizados em junho de 2020 apresentaram tireoglobulina de 39,40 $\mathrm{ng} / \mathrm{mL}$, TSH 0,02 uUl/mL e anticorpo antitireoglobulina (AATG) $21 \mathrm{UI} / \mathrm{mL}$. O paciente foi definido como resistente ao tratamento com radioiodo. Atualmente, encontra-se em acompanhamento, realizando terapia supressiva hormonal com levotiroxina, na dosagem de $200 \mathrm{mcg}$, e com sinais de estabilidade da doença, visualizados na Figura 3. 
Figura 2 - PET/CT. Múltiplos nódulos pulmonares hipermetabólicos, consistentes com lesões secundárias $e$ linfonodomegalia hipermetabólica em cadeia paratraqueal superior direita, de aspecto secundário.

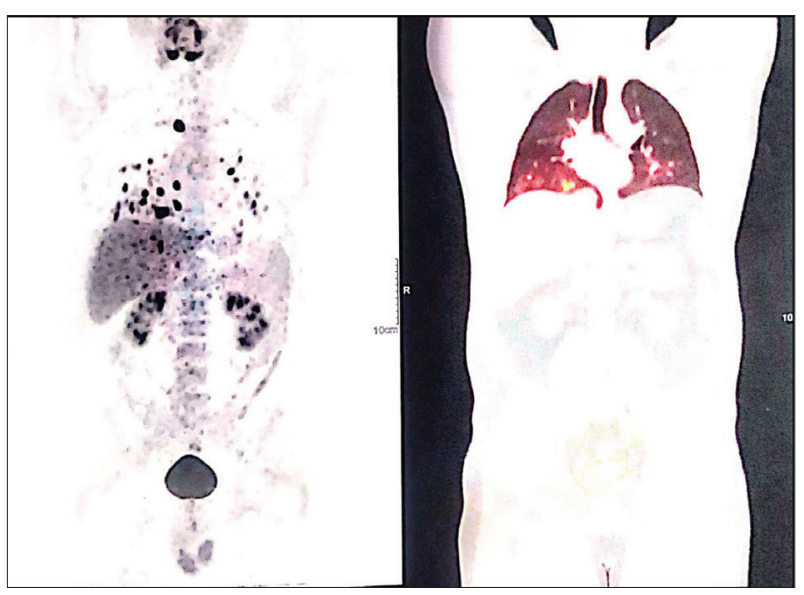

Fonte: Dados da pesquisa.

Realizou-se o exame de imuno-histoquímica para pesquisa de expressão proteica do gene NTRK, utilizando o anticorpo pan-TRK (clone EPR17341), por meio de metodologia automatizada (Ventana BenchMark, Roche e/ou Dako Autostainer Link 48, Agilent), obtendo-se resultado negativo. Para a análise somática do gene $B R A F$, foi realizada a extração do DNA tumoral fixado e impregnado em parafina (FFPE); para preparo da biblioteca, foi utilizada a técnica de reação em cadeia da polimerase (PCR), multiplexado com molecular barcode (QiaSeqDNA V3) e, por fim, foi submetido ao sequenciamento de segunda geração, no qual o resultado apresentou a mutação BRAF V600E no éxon 15.

Figura 3 - PET/CT, a imagem à esquerda foi realizada em julho de 2020, no qual há evidência de baixa atividade metabólica em todas as lesões observadas, sugerindo sinais de estabilidade da doença em comparação com a imagem à direita, realizada em janeiro de 2020.

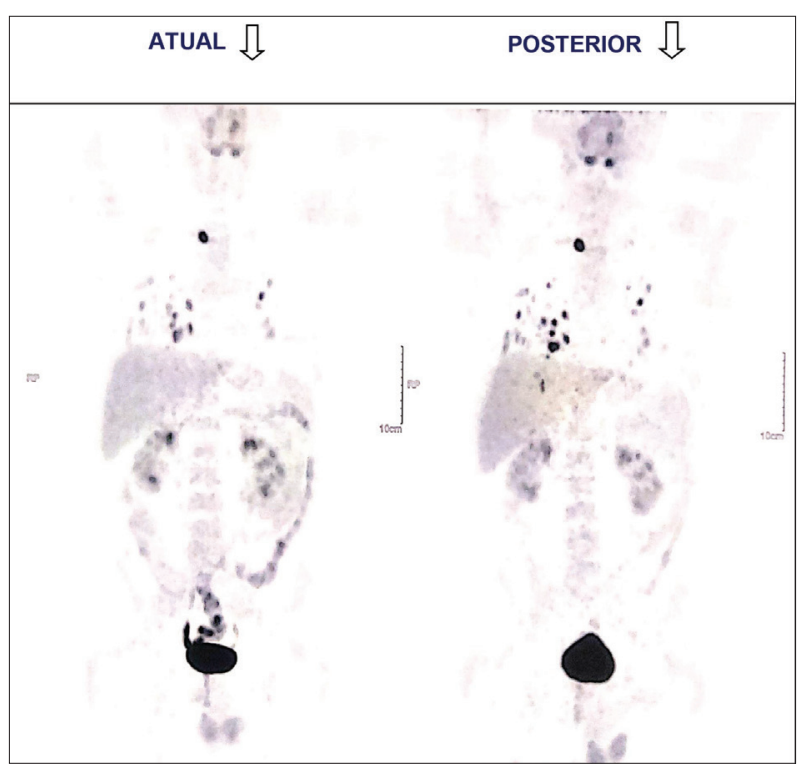

Fonte: Dados da pesquisa.

\section{DISCUSSÃO}

Embora a maioria dos casos de CPT apresentem um prognóstico excelente com uma taxa de sobrevida de 10 anos de mais de $90 \%{ }^{15}$ dos acometidos, as taxas relatadas de recorrência da doença são bastante altas, ocorrendo em $25-35 \%$ dos pacientes ${ }^{16}$. O paciente selecionado para este estudo apresentou persistência da doença, constatada com cerca de um ano após diagnóstico.

Liu et al. ${ }^{17}$ realizaram uma metanálise de 63 estudos, com um total de 20.764 pacientes, para avaliar as associações entre o status da mutação BRAF V600E e características clínico-patológicas agressivas e fatores de pior prognóstico no CPT. A metanálise indicou que a mutação no gene BRAF aumentou o risco de EET em 2,04 vezes e aumentou também o risco de metástase de linfonodo em 1,45 vezes. A taxa de recorrência e persistência da doença também foi duplicada em pacientes com mutação $B R A F$. Além disso, portadores de CPT com mutação BRAF V600E exibiram a taxa de mortalidade 4,61 vezes maior em comparação com os casos sem mutação no gene BRAF. De acordo com os dados da citada metanálise, é evidente a importância da classificação molecular tumoral para melhor conduta terapêutica.

No presente estudo de caso, o paciente apresentou a variante de significado clínico BRAF V600E, sendo esta a alteração genética mais comum associada ao câncer papilar de tireoide clássico. Esta mutação foi associada a pior prognóstico clínico, como recorrência regional e metástases a distância, embora Sherestha et al. ${ }^{6}$ tenham indicado em seu estudo que a mutação BRAF V600E, isoladamente, poderia não apresentar especificidade suficiente para identificar carcinomas papilares com desfechos desfavoráveis.

O termo "carcinoma de tireoide radioiodorefratário (RAIR)" é adotado para casos em que pacientes apresentem baixa captação à radioiodoterapia, que representam $5-15 \%$ dos casos de CPT ${ }^{11}$. De acordo com o estudo de Li et $a l .^{18}$, as taxas de sobrevida em pacientes RAIR são relativamente baixas, de 5 e 10 anos, representando $50 \%$ e $34 \%$, respectivamente. $E$ a taxa de incidência de câncer RAIR no sexo masculino foi de $2,4 \%$, enquanto no sexo feminino foi de $2,1 \%$. Sendo assim, a incidência maior ocorre em pacientes do sexo masculino. $O$ paciente em questão se enquadra nessa definição, pois apresentou ausência de captação do radioiodo nas lesões pulmonares.

Estudo japonês publicado pelo Endocrine Journal avaliou os fatores prognósticos de pacientes com carcinoma diferenciado de tireoide, cursando metástases radioiodorefratárias, e relatou que metástases RAIR em pacientes acima de 60 anos e pacientes do sexo masculino apresentaram riscos acentuados de progressão da doença e menor sobrevida ${ }^{19}$.

Estudo recente realizado por Anekpuritanang et al. ${ }^{11}$, com 60 pacientes, contando com 30 casos RAIR e 30 casos com sucesso na radioiodoterapia, apresentou resultados divergentes de estudos anteriores. Não foi evidenciada 
a relação significativa da mutação $B R A F$ V600E com a sensibilidade ao radioiodo. Embora a mutação BRAF possa alterar a localização e a expressão da proteína NIS, acredita-se que outras características patológicas do tumor poderiam determinar RAIR.

Uma metanálise de 14 estudos, com o total de 2.470 pacientes, demonstrou que a mutação BRAF V600E foi significativamente associada à recorrência do tumor ou à doença persistente e que a prevalência de metástases em linfonodos aumentou em pacientes com mutação $B R A F$ positiva, com uma razão de risco de $1,32^{20}$. Os resultados do presente artigo foram consistentes com os estudos anteriores, pelo fato de a relação da mutação $B R A F$ V600E ter sido favorável a um pior prognóstico clínico, devido à ocorrência da metástase em cinco linfonodos, recorrência da doença, metástase pulmonar e resistência à terapia com radioiodo.

A classificação molecular tumoral prévia pode auxiliar desde a definição da extensão da cirurgia inicial, como no melhor direcionamento terapêutico. $O$ desafio clínico está na identificação precoce daqueles pacientes que precisam de tratamento mais agressivo desde o início e aqueles que terão um curso indolente.

\section{CONCLUSÃO}

Os dados deste relato de caso fornecem evidências de que a mutação no gene BRAF pode estar associada a um pior prognóstico clínico, sendo de grande importância a realização de exames moleculares para identificar essas alterações genéticas e, assim, beneficiar pacientes com terapias mais direcionadas.

\section{REFERÊNCIAS}

1. SEIB, C. D.; SOSA, J. A. Evolving understanding of the epidemiology of thyroid cancer. Endocrinol. Metab. Clin. North Am., Philadelphia, v. 48, n. 1, p. 23-35, Mar. 2019. DOI: 10.1016/j.ecl.2018.10.002.

2. FAGIN, J. A.; WELLS, S. A. JR. Biologic and clinical perspectives on thyroid cancer. N. Engl. J. Med., Boston, v. 375, n. 11, p. 1054-1067, 2016. DOI: 10.1056/NEJMra1501993;

3. THE CANCER GENOME ATLAS RESEARCH NETWORK: Integrated genomic characterization of papillary thyroid carcinoma. Cell, [s.l.], v.159, n. 3, p. 676-690, 2014. DOI:10.1016/jcell.2014.09.050

4. HAY, I. D. et al. Papillary thyroid carcinoma managed at the Mayo Clinic during six decades (1940-1999): temporal trends in initial therapy and long-term outcome in 2444 consecutively treated patients. World J. Surg., New York, n. 26, p. 879-885, 2002.

5. COCA-PELAZ, A. et al. Papillary thyroid cancer-Aggressive variants and impact on management: A narrative review. Adv Ther., Metuchen, n. 37, p.3112-3128, 2020. DOI: 10.1007/s12325-020-01391-1.

6. SHRESTHA, R.T. et al. Multiple mutations detected preoperatively may predict aggressive behavior of papillary thyroid cancer and guide management: a case report. Thyroid, New York, v.25, n. 12, p. 1375-1378, 2015. DOI:10.1089/thy.2015.0278

7. NIKIFOROV, Y.; NIKIFOROVA, M. Molecular genetics and diagnosis of thyroid cancer. Nat. Rev. Endocrinol., London, n. 7, p. 569-580,2011. DOI: $10.1038 /$ nrendo.2011.142.
8. NIKIFOROV, Y. E. Carcinoma da tireóide: vias moleculares e alvos terapêuticos. Mod. Pathol., Baltimore, n. 21, supl. 2, p. S37-43, May 2008. DOI: 10.1038 / modpathol.2008.10.

9. HADDAD, R. et al. NCCN Guidelines ${ }^{\circledR}$ Insights, thyroid carcinoma, version 2.2018. J. Natl. Compr. Canc Netw., Sudbury, v. 16, n.12, p.1429140, 2018. DOI: 10.6004/jnccn.2018.0089;

10. VALVO, V.; NUCERA, C. Coding molecular determinants of thyroid cancer development and progression. Endocrinol. Metab. Clin. North Am., Philadelphia, v. 48, n. 1, p. 37-59, 2019. DOI: 10.1016/j. ecl.2018.10.003.

11. ANEKPURITANANG. T. et al. The association between radioiodine refractory in papillary thyroid carcinoma, sodium / iodide symporter expression, and BRAFV600E mutation. Onco Targets Ther., [s.I.], n. 14, p. 3959-6389, 2021.

12. XING, M. BRAF mutation in thyroid cancer. Endocr. Relat. Cancer, Woodlands, v. 12, n. 2, p. 245-262, 2005. DOI:10.1677/erc.1.0978;

13. BREHAR, A.C. et al. Genetic and epigenetic alterations in differentiated thyroid carcinoma. Journal of Medicine and Life, [s.I.], v. 6, n. 4, p. 403-408, Dec. 2013.

14. HSIAO, S.J.; NIKIFOROV, Y.E. Molecular approaches to thyroid cancer diagnosis. Endocr. Relat. Cancer, Woodlands, v. 21, n. 5, p. T301-T313, 2014. DOI:10.1530/ERC-14-0166.

15. JIN, M. et al. Genetic profiles of aggressive variants of papillary thyroid carcinomas. Cancers, [s.I.], n. 13, p. 892, 2021. DOI: 10.3390/ cancers13040892.

16. PRETE, A. et al. Update on Fundamental Mechanisms of Thyroid Cancer. Front Endocrinol., [s.I.], n. 11, p. 102, Mar. 2020. DOI: 10.3389 / fendo.2020.00102.

17. LIU, C. et al. Associations between BRAFV600E and prognostic factors and poor outcomes in papillary thyroid carcinoma: a meta-analysis. World J. Surg Onc.,[s.I.], n. 14, p. 241, 2016. DOI: 10.1186/ s12957-016-0979-1..

18. LI, G. et al. Radioiodine refractoriness score: a multivariable prediction model for postoperative radioiodine-refractory differentiated thyroid carcinomas. Cancer Med., Oxford, v. 7, n. 11, p. 54485456. 2018. DOI: $10.1002 / \mathrm{cam} 4.1794$

19. ITO Y. et al. Prognosis and prognostic factors of differentiated thyroid carcinoma after the appearance of metastasis refractory to radioactive iodine therapy. Endocr. J., Tokyo, v. 61, n. 8, p. 821-824, 2014. DOI: 10.1507/endocrj.ej14-0181.

20. TUFANO, R. P. et al. BRAF mutation in papillary thyroid cancer and its value in tailoring initial treatment: a systematic review and meta-analysis. Medicine, [s.I.], v. 91, n. 5, p.274-286, Sept. 2012. DOI: 10.1097/MD.0b013e31826a9c71.

21. AMIN, M. B. et al. (Org). AJCC cancer staging manual. $8^{\text {th }}$. ed. New York: Springer International, 2017. Disponível em: https://www.springer. com/gp/book/9783319406176. Acesso em: 30 July 2021.

22. FRANCIS, G. L. et al. Management guidelines for children with thyroid nodules and differentiated thyroid cancer. Thyroid. New York, v. 25, n. 7, p. 716-759, July 2015.

Submetido em: 19/11/2021

Aceito em: 20/11/2021 